\title{
Fault Tolerant Emergency Control to Preserve Power System Stability
}

Pedersen, Andreas Søndergaard; Richter, Jan H.; Tabatabaeipour, Mojtaba; Jóhannsson, Hjörtur; Blanke, Mogens

Published in:

Control Engineering Practice

Link to article, DOI:

10.1016/j.conengprac.2015.11.004

Publication date:

2016

Document Version

Peer reviewed version

Link back to DTU Orbit

Citation (APA):

Pedersen, A. S., Richter, J. H., Tabatabaeipour, M., Jóhannsson, H., \& Blanke, M. (2016). Fault Tolerant Emergency Control to Preserve Power System Stability. Control Engineering Practice, 53, 151-159.

https://doi.org/10.1016/j.conengprac.2015.11.004

\section{General rights}

Copyright and moral rights for the publications made accessible in the public portal are retained by the authors and/or other copyright owners and it is a condition of accessing publications that users recognise and abide by the legal requirements associated with these rights.

- Users may download and print one copy of any publication from the public portal for the purpose of private study or research.

- You may not further distribute the material or use it for any profit-making activity or commercial gain

- You may freely distribute the URL identifying the publication in the public portal

If you believe that this document breaches copyright please contact us providing details, and we will remove access to the work immediately and investigate your claim 


\title{
Fault Tolerant Emergency Control to Preserve Power System Stability
}

\author{
Andreas S. Pedersen*,a, Jan H. Richter ${ }^{\mathrm{d}}$, Mojtaba Tabatabaeipour ${ }^{\mathrm{c}}$, Hjörtur \\ Jóhannsson $^{\mathrm{b}}$, Mogens Blanke ${ }^{\mathrm{a}}$ \\ ${ }^{a}$ Automation and Control Group at Dept. of Electrical Engineering, Technical University of \\ Denmark, 2800 Kgs. Lyngby, Denmark; emails: \{asped,mb@elektro.dtu.dk\} \\ ${ }^{b}$ Center for Electric Power and Energy at Dept. of Electrical Engineering, Technical \\ University of Denmark, 2800 Kgs. Lyngby, Denmark; email: hjjo@elektro.dtu.dk \\ ${ }^{c}$ Energinet DK, Tonne Kjarsvej 65, 7000 Fredericia, Denmark, email: mta@energinet.dk \\ ${ }^{d}$ Siemens AG, Industry Sector, Gleiwitzer Str. 555, 90475 Nuremberg, Germany; email: \\ janrichter@siemens.com
}

\begin{abstract}
This paper introduces a method for fault-masking and system reconfiguration in power transmission systems. The paper demonstrates how faults are handled by reconfiguring remaining controls through utilisation of wide-area measurement in real time. It is shown how reconfiguration can be obtained using a virtual actuator concept, which covers Lure-type systems. The paper shows the steps needed to calculate a virtual actuator, which relies on the solution of a linear matrix inequality. The solution is shown to work with existing controls by adding a compensation signal. Simulation results of a benchmark system show ability of the reconfiguration to maintain stability.
\end{abstract}

Keywords: fault-tolerant control, power system stabiliser, Lure system, actuator faults, wide-area control

\section{Introduction}

Interconnected power systems often experience problems related to lowfrequency electromechanical oscillations (in the $0.1-2 \mathrm{~Hz}$ range). These oscillations arise from the power and phase-angle relationship interacting with generators' inertia, forming an equivalent to a multi-mass-spring system. Large-scale power systems exhibit both local and inter-area eigenmodes. Local eigenmodes are related to those of a single machine against the rest of the system, interarea modes are formed by one group of generating units working against another group. If the eigenmodes are poorly damped, this might lead to a loss of synchronism between synchronous generators and cause cascading of tripping events.

\footnotetext{
${ }^{*}$ Tel.: +45 45253572, Fax: +45 45881799
} 
Power system stabilisers (PSSs) and Power Oscillation Dampers (PODs) (hereafter, collectively termed stabilisers) are effective tools to damp such lowfrequency oscillations. Stabilisers are installed on voltage and power-flow controlling devices to compensate for oscillations in active power transmission [1]. On voltage regulators, the PSS superimposes auxiliary signals on the voltage regulation. The performance of a power system is usually analysed by checking the eigenproperties, and improved by adding active damping control to the electromechanical modes.

The performance of locally designed stabilisers can be improved using widearea measurement signals and wide-area control (WAC) (see e.g. [2]). With the growing use of new technologies such as phasor measurement units (PMU) and fast communication technologies, WAC has given rise to new possibilities in power system operation. This includes use of such wide-area information for improved stability and for emergency control [3]. Furthermore, the communication network allows the use of multiple measurements, whereby fewer devices need to be implemented in a power system to achieve proper damping. When the stabilisers in a multi-machine power system work collaboratively, a proper functionality is expected from each individual stabiliser as a fault in one stabiliser could cause unsatisfactory performance or even instability of the collective control objective. In the present systems, cascaded tripping is a concern if a power damping device is disconnected from the system. In this paper, it is shown shown how wide-area measurement signals can be used and design a wide-area reconfiguration block that can reconfigure the control action and stabilise the system in an event of failure which removes local stabilisers.

With the penetration of synchronised Power Measurements Unit (PMU) technology into power transmission systems, wide-area control has become realistic, not only for normal operation, but in particular during emergency conditions, where reconfiguration schemes can be employed to encapsulate local failures of devices. The purpose of reconfiguring after a fault is to preserve specific properties of the closed-loop system [4]. This work is focused on faults related to devices with stabilisers. Handling of actuator faults to preserve certain properties before and after a fault is referred to as model matching. Model matching design to handle actuator faults were dealt with by [5], [6], who suggested a robust control mixer concept, and [7], who proposed the virtual actuator approach. [8] showed that control reconfiguration of a linear system after an actuator fault is equivalent to disturbance decoupling. Control reconfiguration methods using virtual actuators and sensors for piecewise affine systems and Hammerstein-Wiener systems were proposed in [9], [10], [11] and [12]. AFTC for Lur'e systems with Lipschitz continuous nonlinearity subject to actuator fault using a virtual actuator was presented in [13], where it was assumed that the state of the faulty system is measurable. AFTC for a system with additive Lipschitz nonlinearity subject to actuator faults using a virtual actuator was presented in [14]. Fault tolerant control of polytopic linear parameter varying (LPV) systems subject to sensor faults using virtual sensor was proposed in [15], where the structure of the nominal controller was assumed to be known. It was further assumed that the nominal controller consists of a state feedback 
combined with an LPV observer. [16] considered the problem of control reconfiguration for continuous-time LPV systems with both sensor and actuator faults and without any assumptions about the structure of the nominal controller. In this context input-to-state stability properties of the reconfigured system were investigated. In [17] the control reconfiguration for discrete-time LPV systems with both sensor and actuator faults were considered and both stability and performance of the reconfiguration block was investigated. Reconfigurable control design using a reconfiguration block for input-affine nonlinear dynamical systems was investigated in [18] . Using incremental stability properties, it was shown how to design the nonlinear virtual actuator independent of the nominal controller to achieve ISS of the reconfigured closed-loop system. The design of the nonlinear virtual actuator is achieveded using backstepping control. Extension to nonlinear systems was obtained in [12]. Fault accomodation for large-scale interconnected system was achieved in [19], using a distributed AFTC scheme.

The idea of a virtual actuator is to keep the nominal controller in the loop and transform the input signals designed for the nominal plant to signals appropriate for the remaining healthy actuators. The reconfiguration method is applied to power systems with power oscillation damping controllers. When a damping device fails or is separated from the system, a wide-area virtual actuator is designed that restructures the nominal control loop by using the remaining healthy devices to compensate for the active damping that is missing due to the fault. The advantage of this approach is the separation of fault-tolerant control design from nominal control design. Nominal design and tuning can be used for the remaining stabilisers, fault-tolerance is obtained through a reconfiguration block. Furthermore, as the nominal controllers are still in the reconfigured loop, the implicit knowledge from the stabilisers about the closed-loop performance is preserved.

To our knowledge, no previous attempt of wide-area fault compensation in stabilisers has been done before [20]. Design of wide-area stabilisers was pursued in [2], where locale controls were extended with remote measurements to improve observability of inter-area modes. In [21], wide-area information was used in a hierarchical control scheme. A level of fault tolerance was obtained in ([22], [23]) where a robust wide-area controller used mixed $\mathcal{H}_{2} / \mathcal{H}_{\infty}$ output-feedback control. Adaptive stabilisers using wide-area information were designed in [24] and [25]. Compensation for the effect of wide-area control delays was considered in [26], where a predictor was implemented in the control loop. Using flexible AC transmission systems (FACTS) devices in a wide-area control network for power oscillation damping was considered in [27], where a delay margin for the controllers is introduced. In [28] a two-level stabiliser design is shown for the Brazilian 7-bus southern equivalent with time delay. This test system will also be the basis for the case study in this paper.

The contributions of this work are the following: A wide-area fault-tolerant virtual actuator is designed for the power system, which stabilises the system after a fault removes or separates local stabilising devices. The proposed method does not require changes in local controllers but accommodates faults by adding 
signals to their output. This paper also extends the work done in [20] by finding a reconfiguration that minimises damping degradation during fault, and also accounts for transmission delays in a wide-area communication system.

The paper is organised as follows. The background of stabilisers and faulttolerant control is described in section 2, in which the nonlinear nature of the emergency dynamics is also discussed and a Lure form is introduced to enable generic analysis. Section 3 then discusses reconfiguration based on a virtual actuator approach for nonlinear systems and extends virtual actuator-based theory to cope easily with the problem at hand. Section 4 presents a benchmark test system that develops instability when a line with a series-compensating device is tripped and simulations are performed showing successful reconfiguration and recovery of stability using the new approach.

\section{Background}

\subsection{Power system damping control}

Power systems can obtain oscillatory behaviour under certain circumstances related to the transmission line properties between machines, the level of power transmitted, and the control system parameters. Oscillatory behaviour is encountered under conditions of high reactance of the system (transmission and consumers) and high generator outputs. High synchronizing torque is then needed for generators, but the associated high gain in automatic voltage regulation loops causes deteriorated system damping [1]. Additional damping is provided by an auxiliary control loop, which measures signals related to the oscillation of active power, usually the rotor speed deviation. Power damping can also be achieved by the use of other static power-flow control and voltage control devices (FACTS).

Ideally a stabiliser is installed where the dominant electromechanical modes has highest controllability. Stabilisers can also use several inputs to damp multiple swing modes. When a stabilizing device is separated by a fault, the modes they are intended to control will become less damped. Faults that effect the oscillatory behaviour of a power system include: faults on synchronous generators and synchronous condensers; faults on damping FACTS devices; transmission

line faults that separate control devices. In this paper, all these types of faults are considered where both faults in the control channel and changes in the system dynamics are accounted for.

The detection and isolation of these types of faults is a separate issue, that is treated is the literature. To guarantee isolability of each of these types of faults is a subject of considerable interest, and it is not part of the scope off the present paper. The interested reader could consult recent literature, including [29] and [30] who showed how the structural analysis technique known from Fault Diagnosis literature, see e.g. [4], could be systematically applied to obtain fast fault detection and isolation in a power system based on time domain calculations, [31] who suggested a pattern recognition technique applied on spectral energy information and used a combination of filtering and frequency scaling techniques 
to reduce computational load such that this method could be implementable in real time. Dedicated fault detection for inverters connected to the grid was the subject on [32]. The fault detection and isolation techniques are related to protection methods, which have been studied intensively in the literature. The FDI methods offer detection and isolation for more general classes of faults in generators and devices for voltage stabilisation and it is these types of faults we aim to accommodate with the methods dealt with in the present paper.

\subsection{Control reconfiguration}

In the following, the open-loop power system without stabilisers will be denoted $\Sigma_{P}$ and the stabilisers will be denoted $\Sigma_{C}$ such that the closed-loop system becomes $\left(\Sigma_{P}, \Sigma_{C}\right)$. When a fault occurs, the open-loop system changes from $\Sigma_{P}$ to $\Sigma_{P f}$. The concept of fault-hiding using control reconfiguration is shown in Figure 1. After a fault, the controller $\Sigma_{C}$ interconnected to the faulty plant by means of the connections $\mathbf{y}_{c}=\mathbf{y}_{f}$ and $\mathbf{u}_{c}=\mathbf{u}_{f}$ is generally not suitable for controlling the faulty system. In particular, in the case of stabiliser failures, the loop is partially opened. The reconfiguration block $\Sigma_{R}$ will hide the system fault from the controller and regain stability of the closed-loop system.
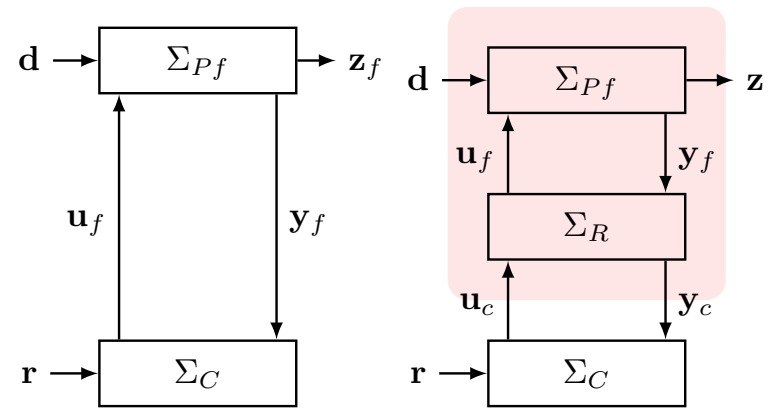

(a) Controller on faulty plant (b) Reconfiguration block hides fault

Figure 1: Illustration of fault hiding. The reconfiguration restructures the nominal control and modifies the output in order to hide fault from the controller.

The reconfiguration block $\Sigma_{R}$ is placed between the faulty plant and the nominal controller, as shown in Figure 1b. Together with the faulty plant, the reconfiguration block $\Sigma_{R}$ forms the reconfigured plant $\Sigma_{P r}=\left(\Sigma_{P f}, \Sigma_{R}\right)$ to which the nominal controller is connected via the signal pair $\left(\mathbf{u}_{c}, \mathbf{y}_{c}\right)$. To enable this, the reconfiguration block must satisfy the following constraint:

Definition 1 (Strict fault-hiding constraint). Consider the nominal system $\Sigma_{P}$ and the faulty system $\Sigma_{P f}$. The reconfigured plant $\Sigma_{P r}$ satisfies the strict faulthiding constraint, if a suitable particular initial condition of the reconfiguration block $\Sigma_{R}$ exists such that the following relation holds:

$$
\forall t \in \mathbb{R}_{+}, \forall \mathbf{d}(t), \forall \mathbf{u}_{c}(t): \mathbf{y}(t)-\mathbf{y}_{c}(t)=0 .
$$


The design of such a reconfiguration block is described in Section 3. The main objective of a reconfiguration is to guarantee stability of the reconfigured system. A secondary objective of the reconfiguration, which minimises the performance degradation, is also introduced.

The performance of stabilisers is often analysed from the eigenproperties of the system. To obtain guarantees for stability during emergency situations, and associated large transients, the normal approach of linear design of stabilisers will not suffice. Instead, a nonlinear model and an adequate nonlinear design approach are required. The performance will still be optimised with regard to the linearised system. The Lure formulation has been used previously on a multi-machine power system to examine the transient behaviour of a system. The general Lure formulation is:

$$
\Sigma_{P}:\left\{\begin{array}{l}
\dot{\mathbf{x}}(t)=\mathbf{A} \mathbf{x}(t)+\mathbf{B}_{v} \mathbf{v}(t)+\mathbf{B u}_{c}(t)+\mathbf{B}_{d} \mathbf{d}(t) \\
\mathbf{v}(t)=\varphi\left(\mathbf{C}_{v} \mathbf{x}(t)\right) \\
\mathbf{y}(t)=\mathbf{C} \mathbf{x}(t) \\
\mathbf{z}(t)=\mathbf{C}_{z} \mathbf{x}(t)
\end{array}\right.
$$

where $A \in \mathbb{R}^{n \times n}, B \in \mathbb{R}^{n \times m}, C \in \mathbb{R}^{r \times n}, C_{v} \in \mathbb{R}^{s \times n}, B_{d} \in \mathbb{R}^{n \times d}$ and $C_{z} \in$ $\mathbb{R}^{n \times q}$. Here $y(t) \in \mathbb{R}^{r}$ is the measured output and $z(t) \in \mathbb{R}^{q}$ is the controlrelevant performance output. The feedback signal $\mathbf{v}(t)$ is obtained using the nonlinear characteristic $\varphi(\cdot): \mathbb{R}^{s} \mapsto \mathbb{R}^{s}$ satisfying the following assumption.

Assumption 1 (Nominal Lure nonlinearity). The function $\varphi$ is decomposed, element-wise Lipschitz, and sector-bounded in the sector $[0, \mathbf{K}]$, with

$\mathbf{K}=\boldsymbol{d i a g}\left(k_{1}, \ldots, k_{s}\right)$, where $k_{1}, \ldots, k_{s}$ defines the sector condition in each element of the nonlinear output $\mathbf{v}$.

The Lure system (1) is controlled by means of a given nominal controller $\Sigma_{C}$. Stabiliser-control strategies usually involve using the generator's angular frequency or the terminal frequency deviation in a supplementary feedback block. The following assumption is made on the nominal closed-loop system.

Assumption 2 (Nominal closed-loop stability). The given nominal closed-loop system of $\Sigma_{P}$ and $\Sigma_{C}$ is input-to-state stable (ISS) ${ }^{1}$ with regard to the inputs $(\mathbf{r}, \mathbf{d})$.

Design techniques for controllers to make Lure systems ISS is well-described in the literature (cf. $[34,35]$ and the references therein).

Faults change the nominal Lure system (1) to the faulty Lure system

\footnotetext{
[33].

${ }^{1} \mathrm{~A}$ system is ISS if functions $\beta \in \mathcal{K} \mathcal{L}, \gamma \in \mathcal{K} \mathcal{K}_{\infty}$ exists such that $|\mathbf{x}(t)| \leq \beta\left(\left|\mathbf{x}^{0}\right|, t\right)+\gamma\left(\|\mathbf{u}\|_{\infty}\right)$
} 


$$
\Sigma_{P f}:\left\{\begin{array}{l}
\dot{\mathbf{x}}_{f}(t)=\mathbf{A}_{f} \mathbf{x}_{f}(t)+\mathbf{B}_{v} \mathbf{v}_{f}(t)+\mathbf{B}_{f} \mathbf{u}_{f}(t)+\mathbf{B}_{d} \mathbf{d}(t) \\
\mathbf{v}_{f}(t)=\varphi_{f}\left(\mathbf{C}_{v} \mathbf{x}_{f}(t)\right) \\
\mathbf{y}_{f}(t)=\mathbf{C x}_{f}(t) \\
\mathbf{z}_{f}(t)=\mathbf{C}_{z} \mathbf{x}_{f}(t)
\end{array}\right.
$$

where all matrices are of the same size as in the non-faulty case. To distinguish the faulty system behaviour from the nominal behaviour, all signals that are affected by faults are labelled by subscript $f$. A stabiliser failure is an event that changes the nominal input matrix $\mathbf{B}$ to the faulty input matrix $\mathbf{B}_{f}$ by setting the corresponding row to zero. The fault is assumed to have been isolated by an existing FDI system, and the necessary mappings in (2) designed from that. The following assumption is made for the faulty system:

Assumption 3 (Stabilisability). The pair $\left(\mathbf{A}_{f}, \mathbf{B}_{f}\right)$ is assumed to be stabilisable.

Although actuator failures (i.e. stabiliser failures) are of primary interest, more general actuator faults are defined. The method presented below is applicable to the following definition of faults.

Definition 2 (Actuator and internal faults). An actuator fault $f$ is an event that changes the nominal input matrix $\mathbf{B} \in \mathbb{R}^{(n \times m)}$ to the faulty input matrix $\mathbf{B}_{f}$ of the same dimensions. An internal fault is an event that changes the system matrix $\mathbf{A}$ to $\mathbf{A}_{f}$, the nominal characteristic $\varphi: \mathbb{R}^{s} \mapsto \mathbb{R}^{s}$ to the faulty characteristic $\varphi_{f}$ of identical dimension and the sector $\mathbf{K}$ to the faulty sector $\mathbf{K}_{f}$.

\section{Wide-area virtual actuator for control reconfiguration}

In this section a new reconfiguration result is presented, using a passivitybased stabilising design of Lure-type systems, extending the result from [13]. The virtual actuator implementation is shown in Figure 2. The signals related to the difference system are labelled by subscript $\Delta$. Define the matrices $\mathbf{A}_{\Delta} \triangleq$ $\mathbf{A}-\mathbf{B}_{f} \mathbf{M}$ and $\mathbf{B}_{\Delta} \triangleq \mathbf{B}-\mathbf{B}_{f} \mathbf{N}$. The reconfiguration block $\Sigma_{R}$ proposed in this paper is a Lure virtual actuator:

$$
\Sigma_{R}:\left\{\begin{aligned}
\dot{\mathbf{x}}_{\Delta}(t)=\mathbf{A}_{\Delta} \mathbf{x}_{\Delta}(t)+\left(\mathbf{A}-\mathbf{A}_{f}\right) \mathbf{x}_{f}(t)+\mathbf{B}_{v} \mathbf{v}_{\Delta}(t) \\
\quad+\mathbf{B}_{\Delta} \mathbf{u}_{c}(t) \\
\mathbf{x}_{\Delta}(0)=\mathbf{x}_{\Delta 0} \\
\mathbf{v}_{\Delta}(t)=\varphi\left(\mathbf{C}_{v}\left(\mathbf{x}_{\Delta}(t)+\mathbf{x}_{f}(t)\right)\right)-\varphi_{f}\left(\mathbf{C}_{v} \mathbf{x}_{f}(t)\right) \\
\mathbf{u}_{f}(t)=\mathbf{M} \mathbf{x}_{\Delta}(t)+\mathbf{N} \mathbf{u}_{c}(t) \\
\mathbf{y}_{c}(t)=\mathbf{y}_{f}(t)+\mathbf{C} \mathbf{x}_{\Delta}(t)
\end{aligned}\right.
$$




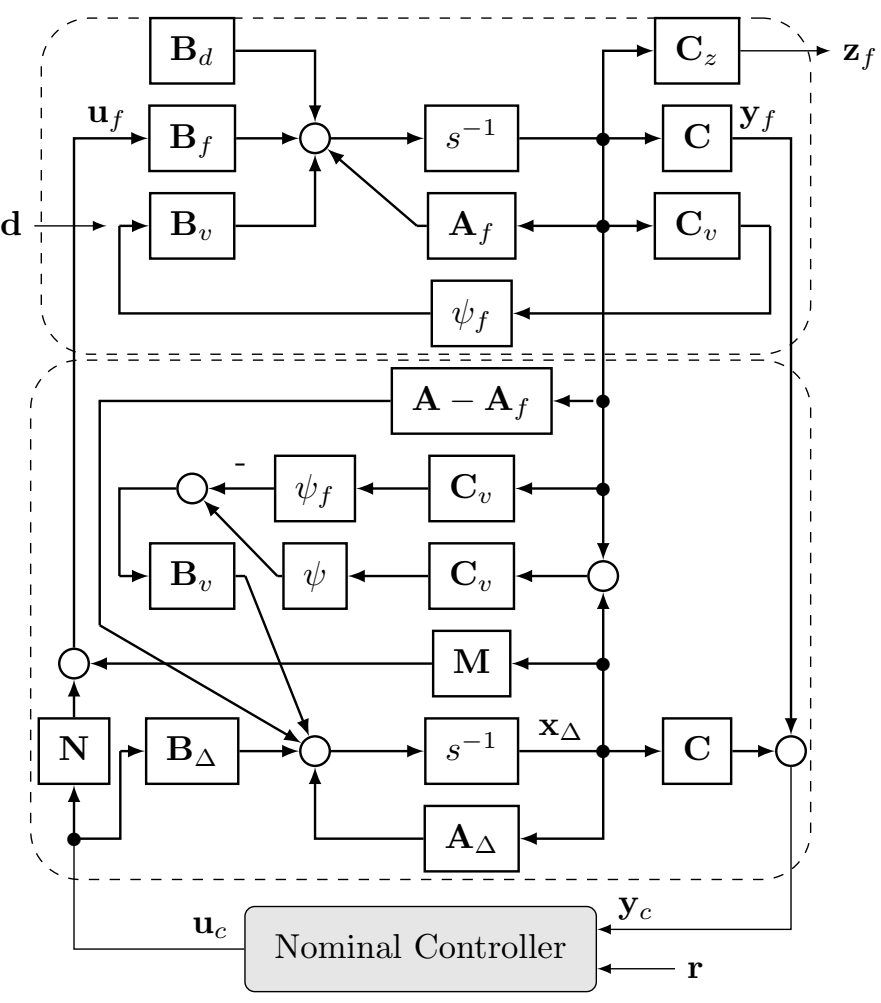

Figure 2: Virtual actuator for Lure-type systems.

The virtual actuator $\Sigma_{R}$, whose linear form was introduced in [7], expresses the difference between nominal and reconfigured dynamics in its state $\mathbf{x}_{\Delta}$ and tries to keep this difference small. The matrices $\mathbf{M}$ and $\mathbf{N}$ are free design parameters that may be used to affect the virtual actuator behaviour. Note that the implementation of the Lure virtual actuator requires the knowledge of the state $\mathbf{x}_{f}$ of the faulty Lure system, which must either be measured or estimated using an observer (observer design for Lure systems is described e.g. in $[36,37])^{2}$. If state information cannot be obtained, internal faults cannot be handled.

In this paper, faults are assumed to appear abruptly and remain effective once they have occurred.

\footnotetext{
${ }^{2}$ The preservation of stability after introducing an observer is expected but must be analysed separately; a generic discussion of the combination of nonlinear virtual actuators with nonlinear observers is available in [12].
} 


\subsection{Fault-hiding property and separation principle}

In order to prove the strict fault-hiding constraint, the state transformation $\mathbf{x}_{f}(t) \rightarrow \tilde{\mathbf{x}}(t) \triangleq \mathbf{x}_{f}(t)+\mathbf{x}_{\Delta}(t)$ is applied, after which the reconfigured plant (2), (3) is described by:

$$
\begin{aligned}
\left(\begin{array}{c}
\dot{\tilde{\mathbf{x}}}(t) \\
\dot{\mathbf{x}}(t)
\end{array}\right)= & \left(\begin{array}{cc}
\mathbf{A} & 0 \\
0 & \mathbf{A}_{\Delta}
\end{array}\right)\left(\begin{array}{c}
\tilde{\mathbf{x}}(t) \\
\mathbf{x}_{\Delta}(t)
\end{array}\right)+\left(\begin{array}{c}
\mathbf{B} \\
\mathbf{B}_{\Delta}
\end{array}\right) \mathbf{u}_{c}(t) \\
& +\left(\begin{array}{c}
\mathbf{B}_{v} \tilde{\mathbf{v}}(t) \\
\mathbf{B}_{v} \mathbf{v}_{\Delta}(t)
\end{array}\right)+\left(\begin{array}{c}
\mathbf{B}_{d} \\
0
\end{array}\right) \mathbf{d}(t) \\
\tilde{\mathbf{x}}(0)= & \mathbf{x}_{0}+\mathbf{x}_{\Delta}, \mathbf{x}_{\Delta}(0)=\mathbf{x}_{\Delta} \\
\tilde{\mathbf{v}}(t)= & \varphi\left(\mathbf{C}_{v} \tilde{\mathbf{x}}(t)\right) \\
\mathbf{v}_{\Delta}(t)= & \varphi\left(\mathbf{C}_{v} \tilde{\mathbf{x}}(t)\right)-\varphi_{f}\left(\mathbf{C}_{v}\left(\tilde{\mathbf{x}}(t)-\mathbf{x}_{\Delta}(t)\right)\right) \\
\mathbf{y}_{c}(t)= & \mathbf{C} \tilde{\mathbf{x}}(t), \mathbf{z}_{f}(t)=\mathbf{C}_{z} \tilde{\mathbf{x}}(t)-\mathbf{C}_{z} \mathbf{x}_{\Delta}(t) .
\end{aligned}
$$

This model shows that $\mathbf{y}_{c}$, the measured output made available to the controller, depends only on the state $\tilde{\mathbf{x}}$, which is governed by the nominal dynamics if the virtual actuator initial condition is $\mathbf{x}_{\Delta}=0$, which proves that the Lure virtual actuator satisfies the strict fault-hiding constraint. Due to Assumption 2, the interconnection $\left(\Sigma_{\tilde{P}}, \Sigma_{C}\right)$ is ISS.

The difference state variable $\mathbf{x}_{\Delta}$ is, as seen from (4), affected by the dynamics of the state variable $\tilde{\mathbf{x}}$ through the variable $\mathbf{v}_{\Delta}$, but not the converse, which would contradict fault hiding. The nominal closed-loop system $\left(\Sigma_{\tilde{P}}, \Sigma_{C}\right)$ is connected in series to the difference system $\Sigma_{R}$, which implies that the series interconnection theorem for input-to-state stable systems is applicable, where the first system $\Sigma_{\tilde{P}}$ is ISS by Assumption 2. It must also be ensured, through proper design, that the difference system is ISS with regard to the inputs $\mathbf{u}_{c}(t)$ and $\tilde{\mathbf{x}}(t)$.

\subsection{Passivity-based stability recovery}

Sufficient conditions for input-to-state stability of the difference system $\Sigma_{R}$ with regard to its external inputs must be given.

Theorem 1 (Global reconfigured closed-loop ISS). Consider the faulty Lure system (2) under Assumptions 2, 1, and let $\mathbf{S}=\mathbf{K}_{f}^{-1}$. The reconfigured closedloop system is globally ISS if $\mathbf{X}=\mathbf{X}^{T} \succ 0$ and $\mathbf{Y}$ of appropriate dimensions exists such that the matrix inequality

$$
\left(\begin{array}{cc}
-\left(\mathbf{X A}^{T}+\mathbf{A X}-\mathbf{B}_{f} \mathbf{Y}-\mathbf{Y}^{T} \mathbf{B}_{f}^{T}\right) & -\mathbf{X} \mathbf{C}_{v}^{T}-\mathbf{B}_{v} \\
\star & \mathbf{S}+\mathbf{S}^{T}
\end{array}\right) \succ 0
$$

is satisfied, where $\mathbf{M}=\mathbf{Y X}^{-1}$.

Proof. We first consider the unforced difference system (for $\mathbf{u}_{c}=0, \tilde{\mathbf{x}}=0$ ) and show that satisfaction of LMI (5) implies global asymptotic stability of 
the difference system. According to the circle criterion, the unforced difference system is absolutely stable at the origin if its linear subsystem is passive, which is the case according to [38] if the matrix inequality

$$
\left(\begin{array}{cc}
-\left(\mathbf{A}-\mathbf{B}_{f} \mathbf{M}\right)^{T} \mathbf{P}-\mathbf{P}\left(\mathbf{A}-\mathbf{B}_{f} \mathbf{M}\right) & -\mathbf{C}_{v}^{T}-\mathbf{P B}_{v} \\
\star & \mathbf{S}+\mathbf{S}^{T}
\end{array}\right) \succ 0
$$

is feasible in the variables $\mathbf{P}=\mathbf{P}^{T} \succ 0$ and $\mathbf{M}$. Terms denoted $\star$ will be induced by symmetry. The latter inequality is nonlinear for the purpose of designing $\mathbf{M}$ due to products between variables $\mathbf{P}$ and $\mathbf{M}$. The following standard trick turns it into an equivalent LMI: the Schur lemma turns it into the equivalent inequalities $\mathbf{S}+\mathbf{S}^{T} \succ 0$ and $-\left(\mathbf{A}^{T} \mathbf{P}+\mathbf{P A}-\mathbf{M}^{T} \mathbf{B}_{f}^{T} \mathbf{P}-\mathbf{P B} \mathbf{B}_{f} \mathbf{M}\right)-\left(\mathbf{C}_{v}^{T}-\mathbf{P} \mathbf{B}_{f}\right)(\mathbf{S}+$ $\left.\mathbf{S}^{T}\right)^{-1}\left(\mathbf{C}_{v}^{T}-\mathbf{P B}_{f}\right)^{T} \succ 0$. Pre- and post-multiplying with $\mathbf{P}^{-1}$ (a congruence transformation) and substitutions $\mathbf{X} \triangleq \mathbf{P}^{-1}$ and $\mathbf{Y} \triangleq \mathbf{M} \mathbf{P}^{-1}$ give the result $-\left(\mathbf{X} \mathbf{A}^{T}+\mathbf{A X}-\mathbf{Y}^{T} \mathbf{B}_{f}^{T}-\mathbf{B}_{f} \mathbf{Y}\right)-\left(\mathbf{X C}_{v}^{T}-\mathbf{B}_{f}\right)\left(\mathbf{S}+\mathbf{S}^{T}\right)^{-1}\left(\mathbf{C}_{v}^{T} \mathbf{X}-\mathbf{B}_{f}\right)^{T} \succ 0$. Applying the Schur lemma once more gives the LMI (5).

It remains to be shown that absolute stability of the unforced difference system extends to the input-to-state stability of the difference system with nonzero inputs $\mathbf{u}_{c}$ and $\tilde{\mathbf{x}}$. This follows from the fact that LMI (5) implies not only global asymptotic stability for all Lure nonlinearities in the sector, but also global exponential stability. Together with Assumptions 2 and 1 and according to [33, Lemma 4.6], this implies that the forced difference system is globally ISS with regard to $\mathbf{u}_{c}$ and $\tilde{\mathbf{x}}$ as inputs.

\subsection{Performance recovery}

The stabilizing reconfiguration found from Theorem 1 is strictly a feasibility problem with an infinite number of solutions. The purpose of stabilisers is to improve damping of lightly damped electromechanical modes in the system, and an obvious objective of the reconfiguration is to minimise the degradation of the reconfigured system compared to the nominal system. The simplest way to incorporate performance goals into the design consists in ignoring the Lure nonlinearity for the purpose of performance optimization (setting it to zero). With this, linear performance indices can be included in the design. Optimal performance is not really achieved for the Lure system, but improvements may, in practice, be found over a purely stabilising design. And in any case, the performance of stabilisers is usually done by checking the eigenproperties of the system Jacobian. Absolute stability is in any case preserved by this semiheuristic design extension.

Performance recovery is defined as follows:

Definition 3 (Stable optimal trajectory recovery). Let $\Sigma_{R}^{*}$ and $\Sigma_{R}$ be two reconfiguration blocks, which stabilises the faulty closed-loop system. The reconfiguration block $\Sigma_{R}^{*}$ optimally approximates the stable trajectory recovery goal if for any $\mathbf{x}_{0}$ it follows that $\forall \mathbf{u}_{c}:\left\|\mathbf{z}-\mathbf{z}_{f}^{*}\right\|_{\mathcal{L} 2} /\left\|u_{c}\right\|_{\mathcal{L} 2}<\left\|\mathbf{z}-\mathbf{z}_{f}\right\|_{\mathcal{L} 2} /\left\|\mathbf{u}_{c}\right\|_{\mathcal{L} 2}$. 
Define the transfer functions $T_{\mathbf{u}_{c} \rightarrow \mathbf{z}_{\Delta}}(s)=\mathbf{C}_{z}\left(s \mathbf{I}-\mathbf{A}_{\Delta}\right)^{-1} \mathbf{B}_{\Delta}$ and $T_{\mathbf{u}_{c} \rightarrow \mathbf{u}_{f}}(s)=$ $\mathbf{M}\left(s \mathbf{I}-\mathbf{A}_{\Delta}\right)^{-1} \mathbf{B}_{\Delta}+\mathbf{N}$, and let $\gamma_{z}$ and $\gamma_{u}$ be

$$
\begin{aligned}
\gamma_{z} & =\min _{\mathbf{M}, \mathbf{N}}\left\|T_{\mathbf{u}_{c} \rightarrow \mathbf{z}_{\Delta}}(s)\right\|_{\infty} \\
\gamma_{u} & =\min _{\mathbf{M}, \mathbf{N}}\left\|T_{\mathbf{u}_{c} \rightarrow \mathbf{u}_{f}}(s)\right\|_{\infty}
\end{aligned}
$$

Finding a reconfiguration that recovers the performance capabilities with regard to definition 3 was found in [39], which was shown to be the solution to the optimization problem (6). A multi-objective reconfiguration synthesis is also presented, where a compromise between recovery and input amplification is used. In an LMI formulation, this can be solved by:

$$
\begin{aligned}
\min _{\mathbf{X} \succ 0, \mathbf{Y}, \mathbf{N}} \lambda \gamma_{z}+(1-\lambda) \gamma_{u} & \\
\text { s.t. } & {\left[\begin{array}{ccc}
\mathbf{X} \mathbf{A}^{T}+\mathbf{A X}-\mathbf{Y}^{T} \mathbf{B}_{f}^{T}-\mathbf{B}_{f} \mathbf{Y} & \mathbf{B}-\mathbf{B}_{f} \mathbf{N} & \mathbf{P C}_{z}^{T} \\
\star & -\gamma_{z} \mathbf{I} & 0 \\
\star & \star & -\gamma_{z} \mathbf{I}
\end{array}\right] \prec 0 } \\
& {\left[\begin{array}{ccc}
\mathbf{X A} \mathbf{A}^{T}+\mathbf{A X}-\mathbf{Y}^{T} \mathbf{B}_{f}^{T}-\mathbf{B}_{f} \mathbf{Y} & \mathbf{B}-\mathbf{B}_{f} \mathbf{N} & \mathbf{Y}^{T} \\
\star & -\gamma_{u} \mathbf{I} & \mathbf{N}^{T} \\
\star & \star & -\gamma_{u} \mathbf{I}
\end{array}\right] \prec 0 }
\end{aligned}
$$

By solving (8) along with (5), a stabilizing reconfiguration that locally recovers the nominal trajectory optimally is found. The stabilising design of the Lure virtual actuator (3) is summarised in Algorithm 1.

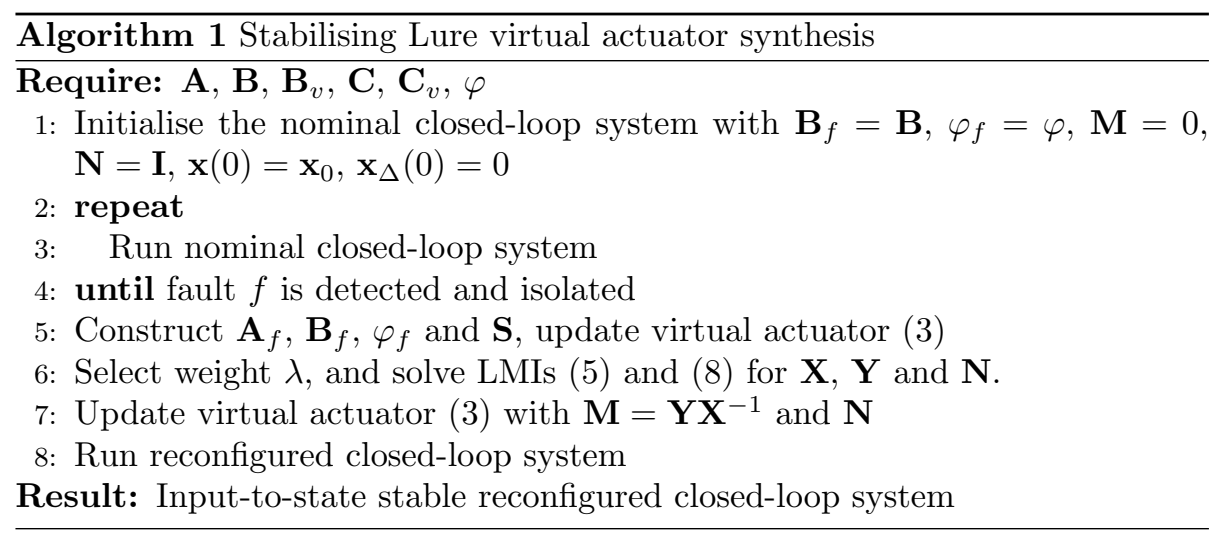

\subsection{Communication delay}

Transmission delays will be present in a wide-area communication system. If the communication delay is much smaller than lowest time period, this can be ignored. However, if the delay is comparable to the electromechanical time periods $(t>0.05 \mathrm{~s})$, the delay will have to be taken into account. Compensation of transmission delays in stabiliser synthesis is a well-studied problem, 
see e.g. [26], [28]. In [26] a prediction method based on Smith's predictor is used to compensate for the time-delay for wide-area stabilisers. In this work the approach of using predictors will also be used, in the case of reconfiguration with time-delayed measurements. Instead of using Smith's predictor scheme, a generic $h$-unit predictor-based approach [40] will be used, as it also allows for compensation of a open-loop unstable system, which could be the case after failures.

We will assume that the communication delay $t_{d}$ is known and constant. In the case of no time-delay, no knowledge other than closed-loop stability need to be known about the controller. However, to design a predictor, the small-signal behaviour of each stabiliser will need to be incorporated into the reconfiguration. Instead of directly using $\mathbf{x}_{\Delta}$ in the reconfiguration compensation, a predictor is introduced:

$$
\begin{aligned}
\mathbf{p}(t) & =e^{\mathbf{A}_{\Delta} t_{d}} \mathbf{x}_{\Delta}\left(t-t_{d}\right)+\int_{-t_{d}}^{0} e^{-\mathbf{A}_{\Delta} \theta} \mathbf{B}_{\Delta} \mathbf{u}_{c}(t+\theta) d \theta \\
\mathbf{u}_{f}(t) & =\mathbf{N u}_{c}(t)+\mathbf{M p}(t) \\
\mathbf{y}_{c}(t) & =\mathbf{y}_{f}(t)+\mathbf{C p}(t)
\end{aligned}
$$

The closed-loop system with a predictor is shown in [40] to preserve damping.

\section{Case study}

In this section, the method will be applied to a benchmark system. A modified seven-bus, five-machine equivalent of the South Brazilian system from [41] is used as a case study. The system has an unstable oscillatory mode, which requires the use of multiple stabilisers, as a single conventional PSS is not able to stabilise it. A thyristor-controlled series compensated (TCSC) line is connected between bus 4 and 6 to provide extra damping. The system is shown in Figure 3.

The TCSC has a stabiliser attached, which uses $\omega_{\text {Itaipu }}$ as input. Locale stabilisers are also attached to the Areia, Santiago and Segredo generators. All loads are considered to be of constant impedance, and the zero-injection buses are removed using Kron reduction.

The machines are represented by flux-decay models with an automatic voltage regulator $(\mathrm{AVR})[42]$.

$$
\begin{aligned}
\dot{\delta}_{i} & =\omega_{i}-\omega_{0} \\
2 H_{i} \dot{\omega}_{i} & =\omega_{0}\left(P_{m i}-E_{q i}^{\prime} I_{q i}-\left(X_{q i}-X_{d i}^{\prime}\right) I_{d i} I_{q i}\right) \\
T_{d 0 i}^{\prime} \dot{E}_{q i}^{\prime} & =E_{f d i}-E_{q i}^{\prime}-\left(X_{d i}-X_{d i}^{\prime}\right) I_{d i} \\
T_{A i} \dot{E}_{f d i} & =-E_{f d i}+K_{A i}\left(V_{\operatorname{ref} i}-V_{i}\right)
\end{aligned}
$$

where $\delta_{i}$ is the rotor angle, $\omega_{i}$ the rotor speed, $E_{q i}^{\prime}$ the quadrature transient voltage, $E_{f d i}$ the field voltage, $\omega_{0}$ the synchronous speed, $H_{i}$ the inertia constant, $P_{m i}$ the mechanical input, $I_{q i}, I_{d i}$ the direct and quadrature axis current, 


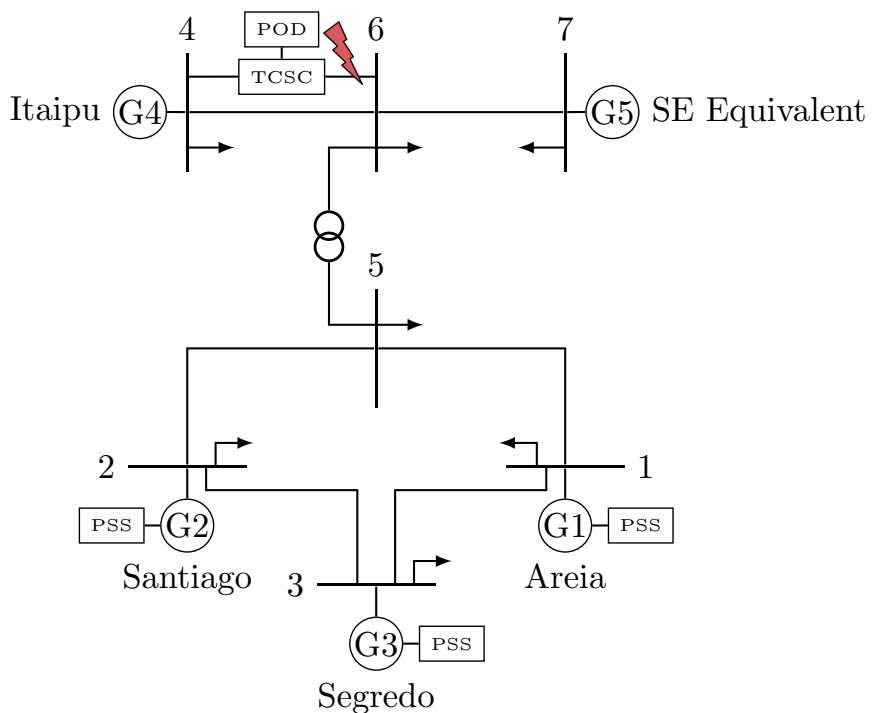

Figure 3: The 7-bus, 5-machine south Brazilian equivalent, where a TCSC line has been inserted between bus $4-6$.

$X_{d i}, X_{q i}, X_{d i}^{\prime}$ the direct, quadrature and direct transient reactance, $T_{d 0 i}^{\prime}$ the direct axis time constant, $T_{A i}$ the AVR time constant, $K_{A i}$ the AVR gain, and $V_{i}$ the terminal voltage magnitude.

The TCSC is modelled as a first-order system.

$$
T_{s} \dot{X}_{\mathrm{tcsc}}=K_{s}\left(X_{\mathrm{tcsc}}^{\mathrm{ref}}-u_{\mathrm{tcsc}}\right)-X_{\mathrm{tcsc}}
$$

where $X_{\mathrm{tcsc}}$ is the TCSC reaction compensation, $T_{s}$ the time constant, $K_{s}$ the TCSC gain, and $X_{\mathrm{tcsc}}^{\mathrm{ref}}$ the reference reactance.

The stator equations are:

$$
\begin{array}{r}
V_{i} \sin \left(\delta_{i}-\theta_{i}\right)-X_{q i} I_{q i}=0 \\
V_{i} \cos \left(\delta_{i}-\theta_{i}\right)-X_{d i}^{\prime} I_{d i}-E_{q i}^{\prime}=0
\end{array}
$$

where $\theta_{i}$ is the terminal voltage angle.

The network equations are:

$$
\begin{aligned}
& I_{d i} V_{i} \sin (\left.\delta_{i}-\theta_{i}\right)+I_{q i} V_{i} \cos \left(\delta_{i}-\theta_{i}\right) \\
&-\sum_{j=1}^{n} V_{i} V_{j}\left(G_{i j} \cos \left(\theta_{i}-\theta_{j}\right)+B_{i j} \sin \left(\theta_{i}-\theta_{j}\right)\right)=0 \\
& I_{d i} V_{i} \cos \left(\delta_{i}-\theta_{i}\right)-I_{q i} V_{i} \sin \left(\delta_{i}-\theta_{i}\right) \\
& \quad-\sum_{j=1}^{n} V_{i} V_{j}\left(G_{i j} \sin \left(\theta_{i}-\theta_{j}\right)-B_{i j} \cos \left(\theta_{i}-\theta_{j}\right)\right)=0
\end{aligned}
$$


where $G_{i j}$ is the conductance and $B_{i j}$ the susceptance.

The algebraic equations are eliminated by solving for the bus voltage in the network equations and inserting them into the stator equations. The resulting currents are solved by inverting the stator equations and inserting them into the dynamics to give a set of ordinary differential equations. To put the system on Lure form, the following assumption is made:

Assumption 4 (State bounds). It is assumed that the quadrature axis internal voltage satisfies $\left|E_{q i}^{\prime}-\overline{E_{q i}^{\prime}}\right| \leq E_{\Delta}$, where $\overline{E_{q i}^{\prime}}$ is the steady-state voltage.

The open-loop system has an unstable electromechanical mode with a damping of $-12.2 \%$ at $0.88 \mathrm{~Hz}$, due to the generator at Itaipu oscillating against the SE equivalent system. The stabilisers are all of the conventional lead-lag type:

$$
C_{\mathrm{pss}}=K_{s} \frac{s T_{W}}{1+s T_{W}}\left(\frac{1+s T_{1}}{1+s T_{2}}\right)^{2}
$$

The closed-loop system is able to stabilise the mode. A time-simulation of the nominal closed-loop system is shown in Figure 4. A disturbance in the power output is rejected by the power system stabilisers.

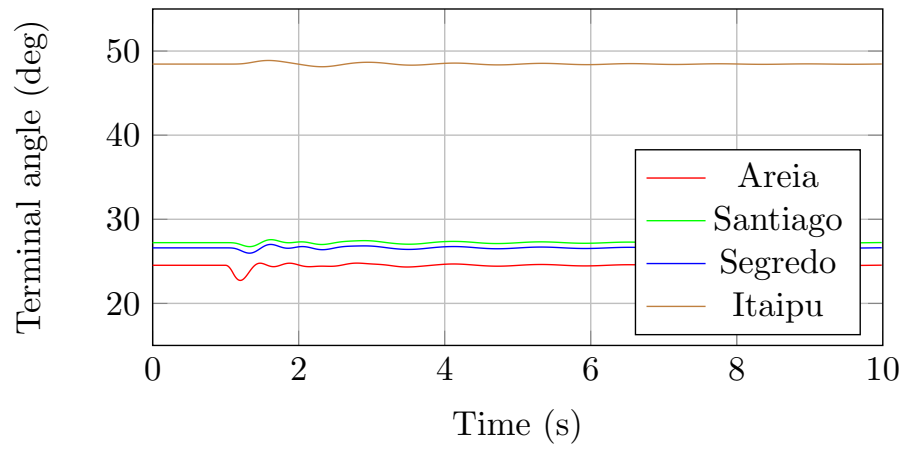

Figure 4: Time simulaton of the closed-loop nominal system.

\subsection{Fault injection}

A faulty situation is simulated in Figure 5 . At the time $t=1 \mathrm{~s}$, a fault happens on the TCSC line connecting bus 4 and 6 . The fault is cleared by tripping the line, which removes the damping near the Itaipu generator. Consequently, the system becomes unstable, as the remaining stabilizing devices on the power system doesn't provide enough damping for the unstable electromechanical mode.

The oscillations will ultimately lead to an angular separation in the power system, which will lead to equipment tripping - or ultimately - a voltage collapse. To avoid this situation, the reconfiguration method will be applied. 


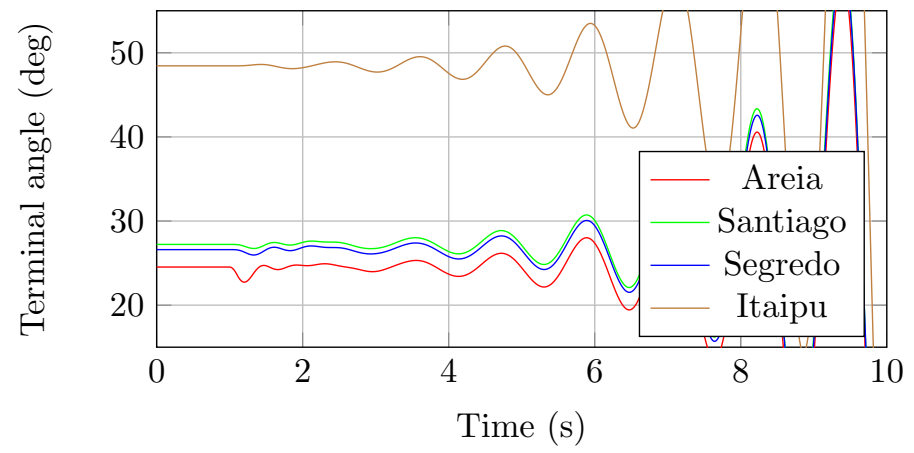

Figure 5: Time simulaton of the closed-loop faulty system, where the compensated line between bus $4-6$ is tripped.

\subsection{Reconfiguration}

A reconfiguration block is introduced to the case study to stabilise the system and provide sufficient damping for the lightly damped modes. The reconfiguration block will superimpose an extra signal on the healthy stabilizing devices, using knowledge about the faulty devices' intended actions. It is assumed that the communication network imposed a signal delay of $t_{d}=0.1 \mathrm{~s}$. It is assumed that an FDI scheme is implemented in the system, which correctly detects and isolates the fault.

The reconfiguration block is calculated from algorithm 1, along with the predictor (9). A $\gamma$ of 0.7 is choosen. A time simulation of the closed-loop reconfigured system is shown in Figure 6.

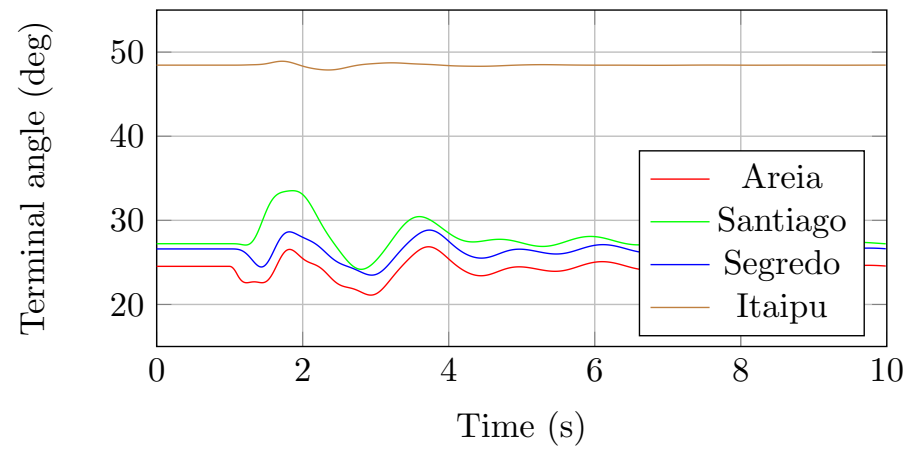

Figure 6: Time simulation of the faulty system, where a reconfiguration block hides the fault from the controller.

The fault-hiding abilities of the reconfiguration make the healthy and removed devices react as if the system is healthy. The control signals from the stabilisers can be seen in Figure 7. The superimposed signal from the reconfiguration - which is the result of the virtual actuator appropriately modifying 
the signal, using knowledge about the power system dynamics - can be seen in Figure 8. The minimally damped electromechanical mode for all scenarios is shown in Table 1.

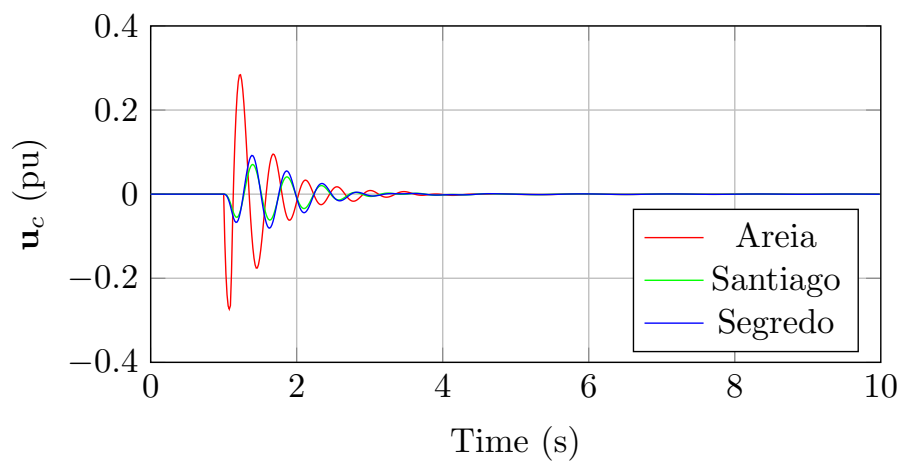

Figure 7: PSS output on the faulty system, where a reconfiguration block hides the fault from the output.

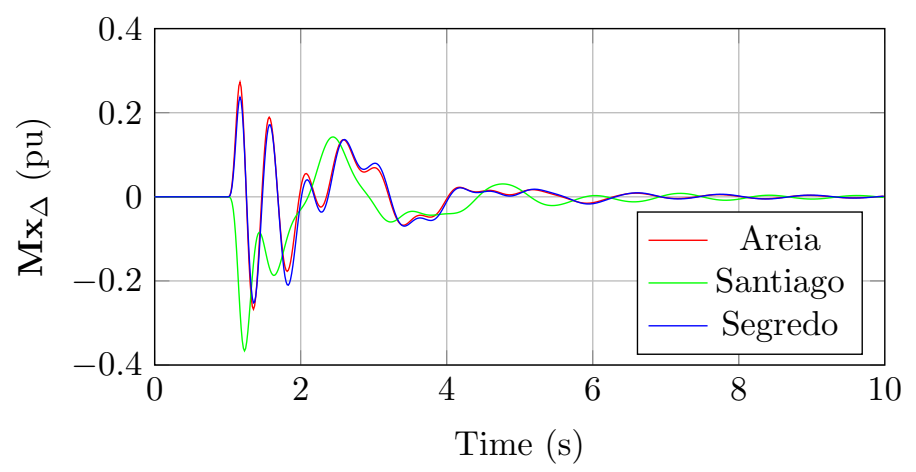

Figure 8: Superimposed signal on the PSS output from the reconfiguration block, which guarantees stability of the closed-loop system and recovers the damping abilities.

\subsection{Computational discussion}

The main computational task in finding the reconfiguration, is the inclusion of the LMI (5), which for very large systems can become large. Various methods can be imployed to decrease to computational time:

- If the system is operated with a contingency list, all reconfigurations can be calculated offline.

- As the problem possesses sparsity (due to each power bus only being connected to a small number of adjacent power busses), sparse methods for semidefinite programming [43] can be employed. 


\begin{tabular}{|ll|}
\hline & $\min \zeta$ \\
\hline Nominal Open-Loop & $-12.2 \%$ \\
Nominal Closed-Loop & $6.38 \%$ \\
Faulty System & $-12.0 \%$ \\
Faulty Reconfigured System & $5.77 \%$ \\
\hline
\end{tabular}

Table 1: System damping ratios (minimum $\% \zeta$ ).

- Existing approaches which is based on aggregation of coherent machines [44] can be used to reduce to problem size.

\section{Conclusion}

In this work, a new design method for virtual actuator fault-tolerant control of Lure systems is introduced and successfully applied to power system reconfiguration. Using the flux-decay model, an optimisation - depending on system parameters - can be performed, which guarantees stability of the reconfigured closed-loop system after a fault.

The salient features of using the fault-hiding method on power systems are:

- The existing control law - which contains valuable implicit knowledge about the electromechanical modes and necessary damping - remains unchanged, while the apparent plant is reconfigured.

- The reconfiguration preserves properties of the preconfigured controller, such as frequency information and wash-out signals, thus leaving the steady-state point unaffected.

- The superimposed reconfiguration signal is added directly to the output of existing controllers, allowing for retrofit in existing power systems.

\section{Acknowledgements}

This work was supported through the SOSPO project by the Danish Council for Strategic Research under grant no. 11-116794.

\section{Bibliography}

[1] P. Kundur, Power System Stability and Control, McGraw-Hill, 1994.

[2] A. F. Snyder, N. Hadjsaid, D. Georges, L. Mili, A. G. Phadke, O. Fawon, W. Sylvain, Inter-Area Oscillation Damping with Power System Stabilizers and Synchronized Phasor Measurements, in: International Conference on Power System Technology, POWERCON '98., 790-794, 1998. 
[3] M. Begovic, D. Novosel, D. Karlsson, C. Henville, G. Michel, Wide-Area Protection and Emergency Control, Proceedings of the IEEE 93 (5) (2005) 876-891.

[4] M. Blanke, M. Kinnaert, J. Lunze, J. Staroswiecki, Diagnosis and FaultTolerant Control, Springer, 3rd edn., 2015.

[5] Z. Yang, M. Blanke, The robust control mixer module method for control reconfiguration, in: Proc. American Control Conference, IEEE Explore, 3407-3411, 2000.

[6] Z. Yang, M. Blanke, M. Verhagen, Robust Control Mixer Method for Reconfigurable Control Design Using Model Matching, IET Control Theory \& Applications 1 (1) (2007) 349-357.

[7] T. Steffen, Control Reconfiguration of Dynamical Systems: Linear Approaches and Structural Tests, vol. 320 of LNCIS, Springer, 2005.

[8] J. Lunze, T. Steffen, Control reconfiguration after actuator failures using disturbance decoupling methods, Automatic Control, IEEE Transactions on 51 (10) (2006) 1590-1601.

[9] J. H. Richter, W. Heemels, N. van de Wouw, J. Lunze, Reconfigurable control of PWA systems with actuator and sensor faults: stability, in: 47th IEEE Conference on Decision and Control, 1060-1065, 2008.

[10] J. H. Richter, J. Lunze, Reconfigurable control of Hammerstein systems after actuator faults, in: Proceedings of the 17th IFAC Word Congress, 3210-3215, 2008.

[11] J. H. Richter, W. Heemels, N. van de Wouw, J. Lunze, Reconfigurable control of piecewise affine systems with actuator and sensor faults: stability and tracking, Automatica 47 (4) (2011) 678-691.

[12] J. H. Richter, Reconfigurable Control of Nonlinear Dynamical Systems - a fault-hiding approach, vol. 408 of LNCIS, Springer, 2011.

[13] J. H. Richter, M. Seron, J. A. De Dona, Virtual actuator for Lure systems with Lipschitz-continuous nonlinearity, in: 8th IFAC Symposium on Fault Detection, Supervision, and Safety for Technical Processes, vol. 8, Mexico City, Mexico, 222-227, 2012.

[14] M. J. Khosrowjerdi, S. Barzegary, Fault tolerant control using virtual actuator for continuous-time Lipschitz nonlinear systems, International Journal of Robust and Nonlinear Control .

[15] S. de Oca, V. Puig, Fault-Tolerant Control design using a virtual sensor for LPV systems, in: 2010 Conference on Control and Fault-Tolerant Systems (SysTol),, 88-93, 2010. 
[16] S. M. Tabatabaeipour, J. Stoustrup, T. Bak, Control Reconfiguration of LPV systems using virtual sensor virtual actuator, in: 8th IFAC Symposium on Fault Detection, Supervision, and Safety for Technical Processes, vol. 8, Mexico City, Mexico, 222-227, 2012.

[17] S. M. Tabatabaeipour, J. Stoustrup, T. Bak, Fault-tolerant control of discrete-time LPV systems using virtual actuators and sensors, International Journal of Robust and Nonlinear Control ISSN 1099-1239, doi: 10.1002/rnc.3194.

[18] S. M. Tabatabaeipour, Galeazzi, Control Reconfiguration of input-affine nonlinear dynamical systems under actuator faults, in: accepter for presentation in 9th IFAC Symposium on Fault Detection, Supervision, and Safety for Technical Processes, Paris, France, 345-352, 2015.

[19] P. Panagi, M. M. Polycarpou, Distributed fault accommodation for a class of interconnected nonlinear systems with partial communication, IEEE Transactions on Automatic Control 56 (12) (2011) 2962-2967.

[20] A. S. Pedersen, J. H. Richter, S. M. Tabatabaeipour, H. Jóhannsson, M. Blanke, Stabiliser Fault Emergency Control using Reconfiguration to Preserve Power System Stability, IFAC 2014 - The 19th World Congress of the International Federation of Automatic Control, Cape Town, South Africa (2014) 9093-9098.

[21] I. Kamwa, R. Grondin, Y. Hebert, Wide-area measurement based stabilizing control of large power systems-a decentralized/hierarchical approach, Power Systems, IEEE Transactions on 16 (1) (2001) 136-153.

[22] H. Chen, Z. Guo, H. Bai, Wide-area Robust H2/Hi Control with pole placement for Damping Inter-area Oscillations, in: IEEE Power Engineering Society General Meeting, 2006., ISBN 1424404932, 7 pp., 2006.

[23] H. Chen, Z. Guo, LMI-based Wide-area Robust Damping Control, 2005 IEEE/PES Transmission \& Distribution Conference \& Exposition: Asia and Pacific (2005) 1-6.

[24] Y. Zhang, G. Chen, O. Malik, G. Hope, An artificial neural network based adaptive power system stabilizer, Energy Conversion, IEEE Transactions on 8 (1) (1993) 71-77.

[25] H. Ni, G. Heydt, R. Farmer, Autonomous damping controller design for power system oscillations, Power Engineering Society Summer Meeting 00 (c) (2000) 1133-1138.

[26] B. Chaudhuri, R. Majumder, B. C. Pal, Wide-area measurement-based stabilizing control of power system considering signal transmission delay, IEEE Transactions on Power Systems 19 (4) (2004) 1971-1979. 
[27] W. Yao, L. Jiang, J. Wen, Q. H. Wu, S. Cheng, Wide-area damping controller of Facts devices for inter-area oscillations considering communication time delays, IEEE Transactions on Power Systems 29 (1) (2014) 318-329.

[28] D. Dotta, A. S. Silva, I. C. Decker, Wide-Area Measurements Based TwoLevel Control Design Considering Signal Transmission Delay, Power Systems, IEEE Transactions on 24 (1) (2008) 1-10.

[29] D. Düstegör, S. Poroseva, M. Hussaini, S. Woodruff, Automated graphbased methodology for fault detection and location in power systems, IEEE Transactions on Power Delivery 25 (2) (2010) 638-646.

[30] T. Knüppel, M. Blanke, J. Østergaard, Fault Diagnosis for Electrical Distribution Systems using Structural Analysis, Int. Journal of Robust and Nonluinear Control 24 (2014) 1446-1465.

[31] K. Krishnanand, P. Dash, M. Naeem, Detection, classification, and location of faults in power transmission lines, International Journal of Electrical Power and Energy Systems 67 (2014) 76-86.

[32] T. Kamel, Y. Biletskiy, L. Chang, Fault diagnosis and on-line monitoring for grid-connected single-phase inverters, Electric Power Systems Research 126 (2015) 68-77.

[33] H. K. Khalil, Nonlinear Systems, Prentice Hall, New Jersey, 3rd edn., 2002.

[34] N. van de Wouw, A. Doris, J. C. A. de Bruin, W. P. M. H. Heemels, H. Nijmeijer, Output-feedback control of Lur'e-type systems with set-valued nonlinearities: a Popov-criterion approach, in: Proc. 2008 American Control Conference, Seattle, WA, USA, 2316-2321, 2008.

[35] J. C. A. de Bruin, A. Doris, N. van de Wouw, W. P. M. H. Heemels, H. Nijmeijer, Control of mechanical motion systems with non-collocation of actuation and friction: a Popov criterion approach for input-to-state stability and set-valued nonlinearities, Automatica 45 (2009) 405-415.

[36] A. Pavlov, N. van de Wouw, H. Nijmeijer, Uniform output regulation of nonlinear systems, Systems \& Control: Foundations and Applications, Birkhäuser, 2006.

[37] B. Brogliato, W. P. M. H. Heemels, Observer design for Lur'e systems with multivalued mappings: a passivity approach, IEEE Trans. Automatic Control 54 (8) (2009) 1996-2001.

[38] S. Boyd, L. E. Ghaouni, E. Feron, V. Balakrishnan, Linear Matrix Inequalities in System and Control Theory, SIAM, 1994.

[39] J. H. Richter, L. Jan, H-based virtual actuator synthesis for optimal trajectory recovery, IFAC Proceedings Volumes (IFAC-PapersOnline) (2009) $1587-1592$. 
[40] T. Furukawa, E. Shimemura, Predictive control for systems with time delay, International Journal of Control 37 (2) (1983) 399-412, ISSN 0020-7179.

[41] N. Martins, L. T. G. Lima, Eigenvalue and frequency domain analysis of small-signal electromechanical stability problems, 1989.

[42] P. W. Sauer, M. A. Pai, Power System Dynamics and Stability, Prentice Hall, 1998.

[43] M. Fukuda, M. Kojima, K. Murota, K. Nakata, Exploiting Sparsity in Semidefinite Programming via Matrix Completion I: General Framework, SIAM Journal on Optimization 11 (3) (2001) 647-674, ISSN 1052-6234.

[44] J. H. Chow, R. Galarza, N. York, W. W. Price, Inertial and slow coherency aggregation algorithms for power system dynamic model reduction, IEEE Transactions on Power Systems 10 (2). 